\title{
Finding General Explicit Formulas for Ising Integral Recursions
}

\author{
D.H. Bailey* J.M. Borwein ${ }^{\dagger}$
}

October 2, 2006

In [2] and [4], we studied integrals of the form

$$
\begin{aligned}
C_{n, k} & :=\frac{4}{n !} \int_{0}^{\infty} \cdots \int_{0}^{\infty} \frac{1}{\left(\sum_{j=1}^{n}\left(u_{j}+1 / u_{j}\right)\right)^{k+1}} \frac{d u_{1}}{u_{1}} \cdots \frac{d u_{n}}{u_{n}} \\
& =\frac{1}{n !} \int_{0}^{\infty} \cdots \int_{0}^{\infty} \frac{d x_{1} d x_{2} \cdots d x_{n}}{\left(\cosh x_{1}+\cdots+\cosh x_{n}\right)^{k+1}} .
\end{aligned}
$$

We also found it useful study normalized variants given by

$$
c_{n, k}=n ! k ! 2^{-n} C_{n, k} .
$$

As it turns out, these integrals can be reduced to one-dimensional integrals by noting that

$$
c_{n, k}=\int_{0}^{\infty} t^{k} K_{0}(t)^{n} d t
$$

where $K_{0}$ is the modified Bessel function. In this form, these integrals can be evaluated to very high precision by means of the tanh-sinh quadrature scheme $[6,1]$, implemented using the ARPREC multiprecision software system [5].

We began the study in [4] by computing $c_{n, k}$ (and thus $C_{n, k}$ ) to very high precision (1000 decimal digits) for all $n \in[2,36]$ and for all $k \in[0,75]$, or in other words a total of 2660 separate 1000-digit quadrature calculations. These computations were performed on the Apple parallel computer system at Virginia Tech. Many of these values (to 500 digit accuracy) are available online at [3].

\footnotetext{
*Lawrence Berkeley National Laboratory, Berkeley, CA 94720, dhbailey@lbl.gov. Supported in part by the Director, Office of Computational and Technology Research, Division of Mathematical, Information, and Computational Sciences of the U.S. Department of Energy, under contract number DE-AC02-05CH11231.

$\dagger^{\dagger}$ Faculty of Computer Science, Dalhousie University, Halifax, NS, B3H 2W5, Canada, jborwein@cs.dal.ca. Supported in part by NSERC and the Canada Research Chair Programme.
} 
In analyzing this data, we made the remarkable discovery, using extensive PSLQ integer relation searches, that for a given $n$, the $\left(C_{n, k}, k \geq 0\right)$ satisfy recursion relations. For instance, for $n=2$ we found

$$
\begin{aligned}
& 0=C_{2,0}-16 C_{2,2} \\
& 0=C_{2,1}-9 C_{2,3} \\
& 0=9 C_{2,2}-64 C_{2,4} \\
& 0=4 C_{2,3}-25 C_{2,5} \\
& 0=25 C_{2,4}-144 C_{2,6},
\end{aligned}
$$

and for $n=3$ we found

$$
\begin{aligned}
& 0=C_{3,0}-84 C_{3,2}+216 C_{3,4} \\
& 0=2 C_{3,1}-69 C_{3,3}+135 C_{3,5} \\
& 0=C_{3,2}-24 C_{3,4}+40 C_{3,6} \\
& 0=32 C_{3,3}-630 C_{3,5}+945 C_{3,7} \\
& 0=125 C_{3,4}-2172 C_{3,6}+3024 C_{3,8} .
\end{aligned}
$$

Similar relations, with larger coefficients, were found for larger $n$.

With some effort, we then were able to identify these patterns for the first few $n$ :

$$
\begin{aligned}
0= & (k+1) C_{1, k}-(k+2) C_{1, k+2} \\
0= & (k+1)^{2} C_{2, k}-4(k+2)^{2} C_{2, k+2} \\
0= & (k+1)^{3} C_{3, k}-2(k+2)\left(5(k+2)^{2}+1\right) C_{3, k+2} \\
& +9(k+2)(k+3)(k+4) C_{3, k+4} \\
0= & (k+1)^{4} C_{4, k}-4(k+2)^{2}\left(5(k+2)^{2}+3\right) C_{4, k+2} \\
& +64(k+2)(k+3)^{2}(k+4) C_{4, k+4} \\
0 \stackrel{?}{=} & (k+1)^{5} C_{5, k}-(k+2)\left(35 k^{4}+280 k^{3}+882 k^{2}+1288 k+731\right) C_{5, k+2} \\
& +(k+2)(k+3)(k+4)\left(259 k^{2}+1554 k+2435\right) C_{5, k+4} \\
& -225(k+2)(k+3)(k+4)(k+5)(k+6) C_{5, k+6} \\
0 \stackrel{?}{=} & (k+1)^{6} C_{6, k}-8(k+2)^{2}\left(7 k^{4}+56 k^{3}+182 k^{2}+280 k+171\right) C_{6, k+2} \\
& +16(k+2)(k+3)^{2}(k+4)\left(49 k^{2}+294 k+500\right) C_{6, k+4} \\
& -2304(k+2)(k+3)(k+4)^{2}(k+5)(k+6) C_{6, k+6} .
\end{aligned}
$$

Here the question mark is used to emphasize the fact that we have no formal proof in the latter two cases.

The presence of factorial-like expressions in the above formulas suggested that these recursions are more naturally cast in terms of the $c_{n, k}$, since these expressions are eliminated by the $k$ ! term in the formula $c_{n, k}=n ! k ! 2^{-n} C_{n, k}$. Indeed, we then found that in terms of $c_{n, k}$ these recursion formulas can be 
written quite compactly as

$$
\sum_{i=0}^{M}(-1)^{i} p_{n, i}(k+i+1) c_{n, k+2 i}=0,
$$

where $M=\lfloor(n+1) / 2\rfloor$. Note, for instance, that the experimental recursions (5) and (6) can be recast compactly in the form of (7) by defining

$$
\begin{array}{ll}
p_{5,0}(x)=x^{6} & p_{6,0}(x)=x^{7} \\
p_{5,1}(x)=35 x^{4}+42 x^{2}+3 & p_{6,1}(x)=x\left(56 x^{4}+112 x^{2}+24\right) \\
p_{5,2}(x)=259 x^{2}+104 & p_{6,2}(x)=x\left(784 x^{2}+944\right) \\
p_{5,3}(x)=225 & p_{6,3}(x)=2304 x .
\end{array}
$$

Table 1 contains some of the other $p_{n, i}$ polynomials that we have discovered experimentally in this way. Our full table of results actually extends up to $n=36$ and $i=18$, which, given the fact that the recursions involve terms spaced by two, is the most that we can obtain from of our set of 2660 quadrature results $\left(c_{n, k}, \quad n \in[2,36], k \in[0,75]\right)$.

Computationally, we found these polynomials by the following procedure. Let $\left(q_{n, k, j}, j=1, M\right)$ denote the relation of length $M$ found by PSLQ in a row of the $c$ array beginning at the entry $c_{n, k}$, so that $\sum_{1 \leq j \leq M} q_{n, k, j} c_{n, k+2 j-2}=0$ to within available numerical precision. We have empirically found that the relation lengths are always of length $M=\lfloor(n+1) / 2\rfloor$. Note that for a given $n$, we only have $q_{n, k, j}$ data for $k \leq 75-2 M$, since the recursions span rows of the $\left(c_{n, k}\right)$ array with a spacing of two, and $k \leq 75$ in the $c$ array. With the $q$ array in hand, we constructed, for a given $n$ and $k$, the $(N+1) \times(N+1)$ matrix

$$
X=\left[\begin{array}{ccccc}
r & \sum_{j=1}^{r} j & \sum_{j=1}^{r} j^{2} & \cdots & \sum_{j=1}^{r} j^{N} \\
\sum_{j=1}^{r} j & \sum_{j=1}^{r} j^{2} & \sum_{j=1}^{r} j^{3} & \cdots & \sum_{j=1}^{r} j^{N+1} \\
\sum_{j=1}^{r} j^{2} & \sum_{j=1}^{r} j^{3} & \sum_{j=1}^{r} j^{r} & \cdots & \sum_{j=1}^{r} j^{N+2} \\
\vdots & \vdots & \ddots & \vdots & \\
\sum_{j=1}^{r} N & \sum_{j=1}^{r} j^{N+1} & \sum_{j=1}^{r} j^{N+2} & \cdots & \sum_{j=1}^{r} j^{2 N}
\end{array}\right]
$$

and the $1 \times(N+1)$ matrix

$$
Y=\left[\begin{array}{c}
\sum_{j=1}^{r} q_{n, k, 1} \\
\sum_{j=1}^{r} j q_{n, k, 2} \\
\sum_{j=1}^{r^{2}} j^{2} q_{n, k, 3} \\
\vdots \\
\sum_{j=1}^{r} j^{N} q_{n, k+N-1}
\end{array}\right]
$$

We then used standard linear system solution routines from the Linpack package (converted to use the ARPREC multiple-precision arithmetic software) to find the solution $A$ of $X A=Y$. Here $N$ is the degree of the polynomial in question (which we typically had to estimate based on earlier results) and $r$ is the number of data points, spaced two apart, that were available in a given row of the $c_{n, k}$ 


\begin{tabular}{|c|c|}
\hline$n$ & $i=1$ \\
\hline 1 & 1 \\
\hline 2 & $4 x$ \\
\hline 3 & $2+10 x^{2}$ \\
\hline 4 & $x\left(12+20 x^{2}\right)$ \\
\hline 5 & $3+42 x^{2}+35 x^{4}$ \\
\hline 6 & $x\left(24+112 x^{2}+56 x^{4}\right)$ \\
\hline 7 & $4+108 x^{2}+252 x^{4}+84 x^{6}$ \\
\hline 8 & $x\left(40+360 x^{2}+504 x^{4}+120 x^{6}\right)$ \\
\hline 9 & $5+220 x^{2}+990 x^{4}+924 x^{6}+165 x^{8}$ \\
\hline 10 & $x\left(60+880 x^{2}+2376 x^{4}+1584 x^{6}+220 x^{8}\right)$ \\
\hline 11 & $6+390 x^{2}+2860 x^{4}+5148 x^{6}+2574 x^{8}+286 x^{10}$ \\
\hline 12 & $x\left(84+1820 x^{2}+8008 x^{4}+10296 x^{6}+4004 x^{8}+364 x^{10}\right)$ \\
\hline$n$ & $i=2$ \\
\hline 3 & 9 \\
\hline 4 & $64 x$ \\
\hline 5 & $104+259 x^{2}$ \\
\hline 6 & $x\left(944+784 x^{2}\right)$ \\
\hline 7 & $816+4752 x^{2}+1974 x^{4}$ \\
\hline 8 & $x\left(9024+17520 x^{2}+4368 x^{4}\right)$ \\
\hline 9 & $5376+54384 x^{2}+52800 x^{4}+8778 x^{6}$ \\
\hline 10 & $x\left(70144+236544 x^{2}+137808 x^{4}+16368 x^{6}\right)$ \\
\hline 11 & $32000+492544 x^{2}+830544 x^{4}+322608 x^{6}+28743 x^{8}$ \\
\hline 12 & $x\left(481280+2469376 x^{2}+2498496 x^{4}+693264 x^{6}+48048 x^{8}\right)$ \\
\hline$n$ & $i=3$ \\
\hline 5 & 225 \\
\hline 6 & $2304 x$ \\
\hline 7 & $7796+12916 x^{2}$ \\
\hline 8 & $x\left(94976+52480 x^{2}\right)$ \\
\hline 9 & $170298+625196 x^{2}+172810 x^{4}$ \\
\hline 10 & $x\left(2409216+2949056 x^{2}+489280 x^{4}\right)$ \\
\hline 11 & $2999076+18232188 x^{2}+11161436 x^{4}+1234948 x^{6}$ \\
\hline 12 & $x\left(48354048+98000448 x^{2}+36003968 x^{4}+2846272 x^{6}\right)$ \\
\hline$n$ & $i=4$ \\
\hline 7 & 11025 \\
\hline 8 & $147456 x$ \\
\hline 9 & $851976+1057221 x^{2}$ \\
\hline 10 & $x\left(13036544+5395456 x^{2}\right)$ \\
\hline 11 & $39605040+106102880 x^{2}+21967231 x^{4}$ \\
\hline 12 & $x\left(683253760+610355200 x^{2}+75851776 x^{4}\right)$ \\
\hline$n$ & $i=5$ \\
\hline 9 & 893025 \\
\hline 10 & $14745600 x$ \\
\hline 11 & $129879846+128816766 x^{2}$ \\
\hline 12 & $x\left(2393358336+791691264 x^{2}\right)$ \\
\hline$n$ & $i=6$ \\
\hline 11 & 108056025 \\
\hline 12 & $2123366400 x$ \\
\hline
\end{tabular}

Table 1: Experimental polynomials $p_{n, i}$ for $1 \leq i \leq 6$ and $1 \leq n \leq 12$. 
array, which number varied with the starting index $k$. These computations were performed using 400-digit arithmetic, both because some of the input coefficients were huge (as large as $10^{40}$ ), and also because of the numerically sensitive nature of the computation - note that the $X$ matrix is the notorious Vandermonde matrix.

Whenever we obtained a satisfactory polynomial, we normalized it so that the coefficients so that the initial coefficient is $(k+1)^{n+1}$, and then expanded each of the terms in a Taylor series about $k+i+1$, so that it would match the formula (7).

We then are faced with the task of compactly representing the data in Table 1. To that end, we empirically discovered that, for all $n$,

$$
\begin{aligned}
& p_{n, 0}(x)=x^{n+1} \\
& p_{n, 1}(x)=\sum_{j=1}^{M} j\left(\begin{array}{c}
n+2 \\
2 j+1
\end{array}\right) x^{n+1-2 j} \\
& =\frac{1}{4}(n+1+x)(x-1)^{n+1}+\frac{1}{4}(x+1)^{n+1}(n+1-x) \\
& p_{n, 2}(x)=\sum_{j=1}^{M-1} \frac{j 4^{j-1}((2 j+3)(n+2)+j+1)}{j+2} \cdot\left(\begin{array}{c}
n+2 \\
2 j+3
\end{array}\right) x^{n-1-2 j} \\
& =\frac{1}{32}\left((n+x+2)^{2}-\frac{7 n}{2}-\frac{11(x+2)}{4}\right)(x-2)^{n+1} \\
& +\frac{1}{32}\left((n-x+2)^{2}-\frac{7 n}{2}+\frac{11(x-2)}{4}\right)(x+2)^{n+1} \\
& -\frac{1}{16} x^{n+1}\left(x^{2}-(n+2)^{2}\right) \\
& \text {... } \\
& \text {... } \\
& p_{n, M}(x)=\left\{\prod_{j=0}^{M}(n-2 j)^{2}\right\} x^{n-2 M} .
\end{aligned}
$$

where $M=\lfloor(n+1) / 2\rfloor$ as before.

We are presently faced with the challenge of finding similar formulas for $i=3,4$ and higher. To that end, let $Q_{n, j, i}$ denote the coefficient of the $j$-th highest term of $p_{n, i}(x)$. For $i=3$ we empirically found (using high-precision polynomial regression on successive right-flank diagonals of the $p_{n, i}$ array) the formulas shown in Table 2 .

Based on the data above, we succeeded in empirically recognizing $\left(R_{j, 0,3}, j \geq\right.$ 0 ), the set of coefficients of the constant (first) terms in the above, by the formula

$$
R_{j, 0,3}=\frac{9^{j+2}\left(17-94(j+2)+16(j+2)^{2}+64(j+2)^{3}\right)-17-10(j+2)}{512(2+(j+2))(5+2(j+2))},
$$

so that $\left(R_{j, 0,3}, j \geq 0\right)=(16 / 9,208 / 5,656,916528 / 105,105728, \cdots)$. However, 


$$
\begin{aligned}
& Q_{n, 1,3}=\left[\frac{16}{9}+\frac{14(n+2)}{3}+\frac{35(n+2)^{2}}{9}\right]\left(\begin{array}{c}
n+2 \\
7
\end{array}\right) \\
& Q_{n, 2,3}=\left[\frac{208}{5}+\frac{528(n+2)}{5}+84(n+2)^{2}\right]\left(\begin{array}{c}
n+2 \\
9
\end{array}\right) \\
& Q_{n, 3,3}=\left[656+1628(n+2)+1254(n+2)^{2}\right]\left(\begin{array}{c}
n+2 \\
11
\end{array}\right) \\
& Q_{n, 4,3}=\left[\frac{916528}{105}+\frac{746304(n+2)}{35}+\frac{240812(n+2)^{2}}{15}\right]\left(\begin{array}{c}
n+2 \\
13
\end{array}\right) \\
& Q_{n, 5,3}=\left[105728+255318(n+2)+189033(n+2)^{2}\right]\left(\begin{array}{c}
n+2 \\
15
\end{array}\right) \\
& Q_{n, 6,3}=\left[\frac{3621152}{3}+\frac{8668640(n+2)}{3}+2112216(n+2)^{2}\right]\left(\begin{array}{c}
n+2 \\
17
\end{array}\right) \\
& Q_{n, 7,3}=\left[\frac{277928800}{21}+\frac{220269736(n+2)}{7}+\frac{478222172(n+2)^{2}}{21}\right]\left(\begin{array}{c}
n+2 \\
19
\end{array}\right) \\
& Q_{n, 8,3}=\left[\frac{1550307040}{11}+\frac{3665920320(n+2)}{11}+239242680(n+2)^{2}\right]\left(\begin{array}{c}
n+2 \\
21
\end{array}\right) \\
& Q_{n, 9,3}=\left[\frac{7340975088}{5}+\frac{17281034682(n+2)}{5}+\frac{12323603619(n+2)^{2}}{5}\right]\left(\begin{array}{c}
n+2 \\
23
\end{array}\right) \\
& Q_{n, 10,3}=\left[\frac{5277350342800}{351}+\frac{4125567389360(n+2)}{117}+\frac{675183110660(n+2)^{2}}{27}\right]\left(\begin{array}{c}
n+2 \\
25
\end{array}\right) \\
& Q_{n, 11,3}=\left[\frac{1063152806288}{7}+\frac{2485457908716(n+2)}{7}+250625779410(n+2)^{2}\right]\left(\begin{array}{c}
n+2 \\
27
\end{array}\right) \\
& Q_{n, 12,3}=\left[\frac{7586040979216}{5}+\frac{17686562513536(n+2)}{5}\right. \\
& \left.+2486774986284(n+2)^{2}\right]\left(\begin{array}{c}
n+2 \\
29
\end{array}\right) \\
& Q_{n, 13,3}=\left[\frac{495551202576928}{33}+\frac{384211149454458(n+2)}{11}\right. \\
& \left.+\frac{807479283683717(n+2)^{2}}{33}\right]\left(\begin{array}{c}
n+2 \\
31
\end{array}\right) \\
& Q_{n, 14,3}=\left[\frac{87747824728470848}{595}+\frac{203676596031279552(n+2)}{595}\right. \\
& \left.+\frac{8367544290284112(n+2)^{2}}{35}\right]\left(\begin{array}{c}
n+2 \\
33
\end{array}\right)
\end{aligned}
$$

Table 2: $Q$ formulas for $i=3$ 
we have not yet been able to find a similar formula for the other two sets of coefficients in the table.

Some results for the case $i=4$ and $i=5$ are given in Table 3 and Table 4 .

We have not yet obtained formulas for $R_{j, k, i}$ for any $i \geq 4$.

\section{References}

[1] David H. Bailey and Jonathan M. Borwein, "Highly parallel, high-precision numerical integration," D-drive Preprint \#294, 2005. Also

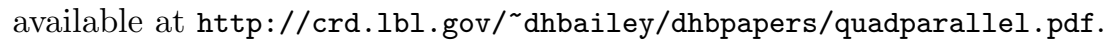

[2] David H. Bailey, Jonathan M. Borwein and Richard E. Crandall, "Integrals of the Ising class," D-drive Preprint \#324, 2006. Also available at http://crd.lbl.gov/ dhbailey/dhbpapers/ising.pdf.

[3] David H. Bailey, Jonathan M. Borwein and Richard E. Crandall, "Ising data," available at

http://crd.lbl.gov/ dhbailey/dhbpapers/ising-data.pdf.

[4] David H. Bailey, Jonathan M. Borwein and Richard E. Crandall, "Hypergeometric forms for Ising-class integrals," available at http://crd.lbl.gov/ dhbailey/dhbpapers/meijer.pdf.

[5] David H. Bailey, Yozo Hida, Xiaoye S. Li and Brandon Thompson, "ARPREC: An Arbitrary Precision Computation Package," 2002, available at http://crd.lbl.gov/ dhbailey/dhbpapers/arprec.pdf.

[6] David H. Bailey, Xiaoye S. Li and Karthik Jeyabalan, "A comparison of three high-precision quadrature schemes," Experimental Mathematics, 14 (2005), 317-329. 


$$
\begin{aligned}
& Q_{n, 1,4}=\left[\frac{48}{5}+\frac{404(n+2)}{15}+28(n+2)^{2}+\frac{35(n+2)^{3}}{3}\right]\left(\begin{array}{c}
n+2 \\
9
\end{array}\right) \\
& Q_{n, 2,4}=\left[\frac{1376}{3}+\frac{3784(n+2)}{3}+1276(n+2)^{2}+\frac{1540(n+2)^{3}}{3}\right]\left(\begin{array}{c}
n+2 \\
11
\end{array}\right) \\
& Q_{n, 3,4}=\left[\frac{70768}{5}+\frac{191672(n+2)}{5}+\frac{189904(n+2)^{2}}{5}+14872(n+2)^{3}\right]\left(\begin{array}{c}
n+2 \\
13
\end{array}\right) \\
& Q_{n, 4,4}=\left[\frac{1077568}{3}+\frac{8654080(n+2)}{9}+\frac{2812576(n+2)^{2}}{3}+\frac{3235232(n+2)^{3}}{9}\right]\left(\begin{array}{c}
n+2 \\
15
\end{array}\right) \\
& Q_{n, 5,4}=\left[\frac{367249408}{45}+\frac{974256128(n+2)}{45}+\frac{312682496(n+2)^{2}}{15}\right. \\
& \left.+\frac{353769728(n+2)^{3}}{45}\right]\left(\begin{array}{c}
n+2 \\
17
\end{array}\right) \\
& Q_{n, 6,4}=\left[\frac{1209630720}{7}+\frac{15929755648(n+2)}{35}+\frac{15185155072(n+2)^{2}}{35}\right. \\
& \left.+\frac{807365632(n+2)^{3}}{5}\right]\left(\begin{array}{c}
n+2 \\
19
\end{array}\right) \\
& Q_{n, 7,4}=\left[\frac{38344335360}{11}+\frac{100406677504(n+2)}{11}+8630992896(n+2)^{2}\right. \\
& \left.+3177865216(n+2)^{3}\right]\left(\begin{array}{c}
n+2 \\
21
\end{array}\right) \\
& Q_{n, 8,4}=\left[\frac{339443941376}{5}+\frac{884613873664(n+2)}{5}+\frac{830889689088(n+2)^{2}}{5}\right. \\
& \left.+60643573760(n+2)^{3}\right]\left(\begin{array}{c}
n+2 \\
23
\end{array}\right) \\
& Q_{n, 9,4}=\left[\frac{351507757137920}{273}+\frac{304134612582400(n+2)}{91}+\frac{65554870435840(n+2)^{2}}{21}\right. \\
& \left.+\frac{7915077304320(n+2)^{3}}{7}\right]\left(\begin{array}{c}
n+2 \\
25
\end{array}\right) \\
& Q_{n, 10,4}=\left[\frac{167449863389184}{7}+\frac{1299541885714432(n+2)}{21}+\frac{402689202847744}{7}(n+2)^{2}\right. \\
& \left.+\frac{434820240637952(n+2)^{3}}{21}\right]\left(\begin{array}{c}
n+2 \\
27
\end{array}\right)
\end{aligned}
$$

Table 3: $Q$ formulas for $i=4$ 


$$
\begin{aligned}
& Q_{n, 1,5}=\left[\frac{256}{3}+\frac{2288(n+2)}{9}+\frac{2684(n+2)^{2}}{9}+\frac{1540(n+2)^{3}}{9}+\frac{385(n+2)^{4}}{9}\right]\left(\begin{array}{c}
n+2 \\
11
\end{array}\right) \\
& Q_{n, 2,5}=\left[\frac{759296}{105}+\frac{743808(n+2)}{35}+\frac{122408(n+2)^{2}}{5}+13728(n+2)^{3}+\frac{10010(n+2)^{4}}{3}\right]\left(\begin{array}{c}
n+2 \\
13
\end{array}\right) \\
& Q_{n, 3,5}=\left[382976+\frac{3339200(n+2)}{3}+1263444(n+2)^{2}+\frac{2086084(n+2)^{3}}{3}\right. \\
& \left.+165165(n+2)^{4}\right]\left(\begin{array}{c}
n+2 \\
15
\end{array}\right) \\
& Q_{n, 4,5}=\left[\frac{244814848}{15}+\frac{6346101632(n+2)}{135}+\frac{7117278752(n+2)^{2}}{135}\right. \\
& \left.+\frac{3857818432(n+2)^{3}}{135}+\frac{179835656(n+2)^{4}}{27}\right]\left(\begin{array}{c}
n+2 \\
17
\end{array}\right) \\
& Q_{n, 5,5}=\left[\frac{64407202304}{105}+\frac{61380724512(n+2)}{35}+\frac{68163514296(n+2)^{2}}{35}\right. \\
& \left.+1042470872(n+2)^{3}+\frac{3588289042(n+2)^{4}}{15}\right]\left(\begin{array}{c}
n+2 \\
19
\end{array}\right) \\
& Q_{n, 6,5}=\left[\frac{5844676718592}{275}+\frac{664345482624(n+2)}{11}+\frac{332618280016(n+2)^{2}}{5}\right. \\
& \left.+\frac{176201471616(n+2)^{3}}{5}+\frac{199562073228(n+2)^{4}}{25}\right]\left(\begin{array}{c}
n+2 \\
21
\end{array}\right) \\
& Q_{n, 7,5}=\left[\frac{6263587389440}{9}+\frac{265634430878272(n+2)}{135}+\frac{290601868592872(n+2)^{2}}{135}\right. \\
& \left.+\frac{152609313286232(n+2)^{3}}{135}+\frac{34194027495122(n+2)^{4}}{135}\right]\left(\begin{array}{c}
n+2 \\
23
\end{array}\right) \\
& Q_{n, 8,5}=\left[\frac{17910569817574400}{819}+\frac{5602496418424960(n+2)}{91}+\frac{1406290263480160(n+2)^{2}}{21}\right. \\
& \left.+\frac{733095709194560(n+2)^{3}}{21}+\frac{69750157030840(n+2)^{4}}{9}\right]\left(\begin{array}{c}
n+2 \\
25
\end{array}\right) \\
& Q_{n, 9,5}=\left[\frac{4662266119508736}{7}+1868250181723728(n+2)+\frac{14155273422548796(n+2)^{2}}{7}\right. \\
& \left.+\frac{7332863764884996(n+2)^{3}}{7}+\frac{1615123753083945(n+2)^{3}}{7}\right]\left(\begin{array}{c}
n+2 \\
27
\end{array}\right)
\end{aligned}
$$

Table 4: $Q$ formulas for $i=5$ 